Arnaud Girardot-Miglierina, Daniel Clerc, Mohammad Alyami, Laurent Villeneuve, Olivia Sgarbura, Marc-André Reymond and Martin Hübner*, on behalf of the ISSPP PIPAC study group

\title{
Consensus statement on safety measures for pressurized intraperitoneal aerosol chemotherapy
}

https://doi.org/10.1515/pp-2021-0125

Received May 5, 2021; accepted September 2, 2021;

published online November 2, 2021

\section{Abstract}

Objectives: Pressurized intraperitoneal aerosol chemotherapy (PIPAC) is a promising treatment for peritoneal cancer that entails, however, potential risks for the caregivers in the operating room (OR). This study aimed to reach a consensus within the PIPAC community on a comprehensive safety protocol.

Methods: Active PIPAC centers were invited to participate in a two-round Delphi process on 43 predefined items: concise summaries of the existing evidence were presented together with questions formulated using the population,

Arnaud Girardot-Miglierina and Daniel Clerc contributed equally to this work.

Collaborators of ISSPP PIPAC Study Group are listed in Appendix section.

*Corresponding author: Prof. Martin Hübner, Department of Visceral Surgery, Lausanne University Hospital (CHUV), Rue du Bugnon 46, 011 Lausanne, Switzerland, E-mail: martin.hubner@chuv.ch Arnaud Girardot-Miglierina and Daniel Clerc, Department of Visceral Surgery, Lausanne University Hospital CHUV, University of Lausanne (UNIL), Lausanne, Switzerland. https://orcid.org/0000-0002-92853312 (D. Clerc)

Mohammad Alyami, Department of General Surgery and Surgical Oncology, Oncology Center, King Khalid Hospital, Najran, Saudi Arabia

Laurent Villeneuve, Department of Public Health, Clinical Research and Epidemiological Unit, Lyon University Hospital, Lyon, France; and University of Lyon, Lyon, France

Olivia Sgarbura, Department of Surgical Oncology, Cancer Institute Montpellier (ICM), Montpellier, France; University of Montpellier, Montpellier, France; and IRCM, Institut de Recherche en Cancérologie de Montpellier, INSERM U1194, Université de Montpellier,

Montpellier, France. https://orcid.org/0000-0002-6965-3697

Marc-André Reymond, Department of General and Transplant Surgery, University Hospital Tübingen and National Center for Pleura and Peritoneum, Tübingen, Germany intervention, comparator, and outcome framework. According to the Grading of Recommendations Assessment, Development, and Evaluation, the strength of recommendation was voted by panelists, accepting a consensus threshold of $\geq 50 \%$ of the agreement for any of the four grading options, or $\geq 70 \%$ in either direction.

Results: Forty-seven out of 66 invited panelists answered both rounds (response rate $76 \%$ ). The consensus was reached for 41 out of 43 items (95.3\%). Strong and weak recommendations were issued for 30 and 10 items, respectively. A positive consensual recommendation was issued to activate laminar airflow without specific strength, neither strong nor weak. No consensus was reached for systematic glove change for caregivers with a high risk of exposure and filtering facepiece mask class 3 for caregivers with low risk of exposure.

Conclusions: A high degree of consensus was reached for a comprehensive safety protocol for PIPAC, adapted to the risk of exposure for the different caregivers in the OR. This consensus can serve as a basis for education and help reach a high degree of adherence in daily practice.

Keywords: education and training; expert consensus; personal protective equipment; PIPAC; safety.

\section{Introduction}

Pressurized intraperitoneal aerosol chemotherapy (PIPAC) has been developed as a new drug delivery system to treat patients with nonresectable peritoneal metastases of various primaries [1-3].

Chemotherapeutic agents (CA) are manipulated during PIPAC. Thus, there is a potential risk of exposure to liquid and aerosolized CA for caregivers present in the operating room (OR) during the procedure [4]. Before the first-inhuman use, and in collaboration with an independent organization certified for occupational health risk assessment (DEKRA Industrials GmbH, Stuttgart, Germany), the pioneer team in Bielefeld performed a detailed risk assessment and developed a dedicated safety protocol. This original safety 
protocol included tightness of the abdomen, laminar airflow ventilation in the $\mathrm{OR}$, controlled aerosol waste, remote control of the procedure, and wearing protective clothing, gloves, and glasses. Simulations of the worst-case scenario (total release of the chemotherapeutic aerosol into the $\mathrm{OR}$ with the person's presence for $30 \mathrm{~min}$ ) calculated an inhaled CA dose between 1:100,000 and 1:1,000,000 of a usual chemotherapeutic dose [5]. The safety protocol was successfully validated under clinical conditions during the first PIPAC procedures with no platin traces detected in the air (detection limit: $0.000009 \mathrm{mg} / \mathrm{m}^{3}$ ). Assuming a platin exposition of $8 \mathrm{~h}$ daily, a maximal substance index $<18 \%$ of the acceptable exposition limit was found, allowing the audit to conclude that the implemented protective measures were meeting the regulatory requirements in Germany (Technische Regeln für Gefahrstoffe [TRGS] 402).

In the following years, multiple PIPAC safety audits were conducted in several European countries [4-12]. In most audits, PIPAC was performed in OR with advanced ventilation OR system meeting the norm ISO 14644-1 class $\leq 5$ but no laminar airflow. In none of these studies, traces of platin were detected in the air $[4,6,7,9,11,13,14]$. However, various degree of contamination of instruments and surfaces was documented $[6,7,9,14,15]$. Assuming thorough implementation of the safety protocols, all independent audits concluded so far that PIPAC can be performed safely, meeting European and national legal and regulatory requirements. Biological monitoring in the blood $[7,16]$ or urine [8] of healthcare workers showed no traces of chemotherapeutic drugs after PIPAC. The German pioneer group implemented a Critical Incident Reporting System for the first 650 PIPAC procedures. Two minor incidents related to disconnection in the tubing system were reported. No severe incident, in particular no leakage of the toxic aerosol, was recorded [5].

In the meantime, PIPAC is diffusing into clinical practice worldwide. Participation in an International Society for the Study of Pleura and Peritoneum (ISSPP) PIPAC training course is required for technology access, and the safety protocols are an essential component of this course [17]. However, a recent survey amongst PIPAC expert centers worldwide showed variable adherence to protective measures [18]. For example, many PIPAC centers now recommend using filtering facepiece (FFP)-2 masks, which were not originally considered necessary by the DEKRA organization. Another recent study on everyday practice and the need for information relating to the risk of exposure suggested that adherence to different protective measures was variable but that the need for continuous education was high [19]. Furthermore, knowledge concerning the risks of $\mathrm{CA}$ and the safety protocol was variable amongst $\mathrm{OR}$ professionals, including surgeons, anesthetists, anesthesia nurses, scrub nurses, and cleaning staff.

Thus, there is a need for establishing a consensus on the required PIPAC safety measures. Therefore, we designed a Delphi study to reach an agreement on a comprehensive safety protocol among active PIPAC centers worldwide.

\section{Materials and methods}

The present study methodology consisted of a two-round Delphi consensus process and was developed in agreement with current standards for developing consensus guidelines [20]. The project was initiated in early 2020 following two surveys on current PIPAC safety practices and perception $[18,19]$. The study was performed under the precepts established by the Declaration of Helsinki.

The authors of this study formed the Guidelines development group (GDG), which consisted of internationally represented surgeons with extensive expertise with PIPAC therapy and developing consensus guidelines. The pioneer German team contributed with its 10-year expertise in occupational health protocols for PIPAC. Four GDG members (MA, LV, OS, and $\mathrm{MH}$ ) are an active part of the educational group of the ISSPP and are in charge of the training curriculum for PIPAC, including its safety aspects [17].

Delphi questions taking into account all different aspects of the topic were defined a priori and formulated according to the population, intervention, comparator, and outcome framework [21], by five members of the GDG (AGM, DC, MA, OS, and MH). For determining the questions, the existing literature was analyzed, looking for the best available evidence from the first description of the PIPAC procedure [3] up to December 2019. Questions were divided into four broad categories: i) personal protective equipment (PPE), ii) environmental protection, iii) prevention of exposure to aerosolized chemotherapy, and iv) general preventive measures. Of note, these questions included items that have not been specified or explicitly mentioned in prior works on occupational health aspects of PIPAC [7-9]. Furthermore, personal protective measures were studied separately for caregivers at high or low risk of exposure, respectively. The definition of caregivers at high or low risk of exposure is outlined in Figure 1 and further defined in the Supplementary Material.

The targeted expert panel included nonselected leaders of all active PIPAC programs identified before December 2019 and previously invited for participation in the development of consensus guidelines for PIPAC technical aspects [22]. No center was deliberately excluded from the process.

An interactive online survey (SurveyMonkey Inc., San Mateo, CA) was sent to the expert panel. Delphi questions were presented together with concise summaries and references to the evidence (Supplementary Material). Experts were asked to provide their level of agreement for, or against, the use of each detailed safety measure and give closed-end recommendations on each item, by the use of a two-sided scale (strong positive, weak positive, weak negative, and strong negative), according to the Grading of Recommendations Assessment, Development, and Evaluation approach [23]. All responders of Delphi's first round were invited to participate in the second round. The second Delphi round presented the same information and questions with additional feedback on the results of the first round. Every participant had one month to 


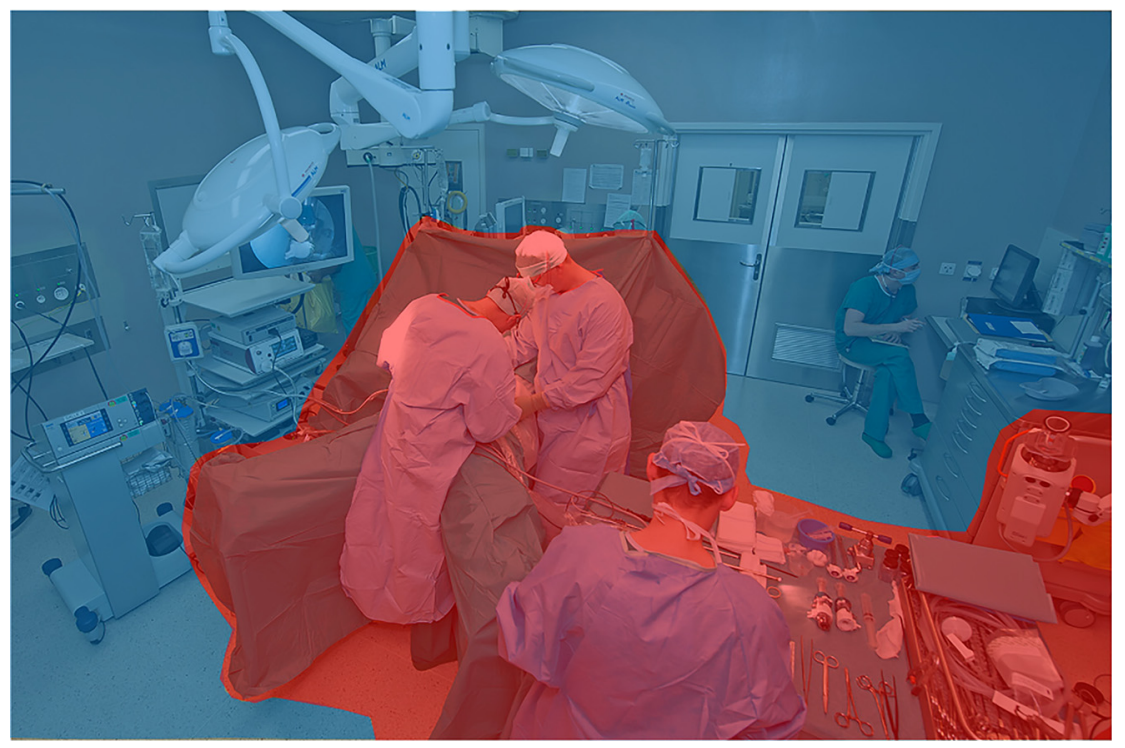

Figure 1: PIPAC procedure operating room scenario.

Definition of high risk vs. low risk for caregivers during PIPAC procedure.

Caregivers at high risk of potential direct exposure to CA (red zone) include surgeons and the surgical team (scrub nurses and surgical assistant), and personnel assigned to the manipulation of the injector. Caregivers at low risk of potential direct exposure to CA (blue zone) include the anesthesiology team, circulators, visitors, and the cleaning staff. Of note, during the remote administration of aerosol chemotherapy and until pneumoperitoneum evacuation, any caregivers entering the operating room are considered at high risk of exposure to aerosolized chemotherapy.

answer the survey, and nonresponders received a minimum of three reminders.

\section{Statistical analysis}

The GDG analyzed the data. Descriptive statistics were used to summarize the results of the expert consensus. The consensus was defined as $\geq 50 \%$ of the agreement for any of the four grading options, or as $70 \%$ agreement for a combined weak or strong recommendation, regardless of the direction, negative or positive.

\section{Results}

There were 66 participants. Response rates for Delphi's first round were $52(87 \%)$, and 47 of these responders completed then Delphi's second round, resulting in a final response rate of $76 \%$. Surgeons represented 46 of the responders completing the entire Delphi, holding a consultant position for 31 (67\%).

The consensus was reached for 41 out of 43 items (95.3\%). In summary, 26 recommendations (60.4\%) were strong positive, while the remainders were either weak positive $(n=9,20.9 \%)$, weak negative $(n=1)$, or strong negative $(\mathrm{n}=4,9.3 \%)$. One recommendation (activation of laminar airflow) reached consensus with $>70 \%$ of combined strong and weak positive agreement. No consensus was reached for two items (4.7\%) after the two Delphi rounds, namely the change of gloves after $30 \mathrm{~min}$ for caregivers with a high risk of exposure and the use of FFP mask class 3 for caregivers with a low risk of exposure.

The following paragraphs provide the synopsis of evidence and degree of consensus divided into four categories of safety measures: (I) PPE, (II) environmental protection, (III) prevention of exposure to aerosolized chemotherapy, and (IV) general preventive measures. Details are provided in Supplementary Material, Appendix 1.

\section{Personal protective equipment (PPE)}

Exposure to CA during PIPAC can occur through direct contact (dermal or ocular) with contaminated surfaces or materials [4]. Several studies examined gloves, hands, devices, injectors, trocars, and floor wiping samples for platin traces and reported highly variable contamination levels $[6,8,9]$. Differences in current practices regarding the PPE required for performing PIPAC have been observed among expert centers [18]. In this Delphi study, there was a large consensus for the PPE needed to perform PIPAC safely. Figure 2 shows the results stratified by the risk of exposure. No agreement was reached for changing gloves every $30 \mathrm{~min}$ (high risk of exposure) instead of keeping the same gloves from the beginning to the end of the procedure. Optimal PPE for caregivers at increased risk of exposure during the PIPAC procedure is summarized in Figure 3. However, there is no transdermal absorption of platin or anthracyclins, and the risk linked to exposition to liquids is probably limited to local cutaneous or ocular toxicity. Biological monitoring studies confirmed the efficacy of PPE: no platin traces were found in blood and urine samples of persons performing PIPAC regularly [8, 9], even after 1,200 procedures [16].

\section{Environmental protection}

The degree of consensus for the different measures for environmental protection is detailed in Figure 4. Among 
For caregivers with high risk of exposure

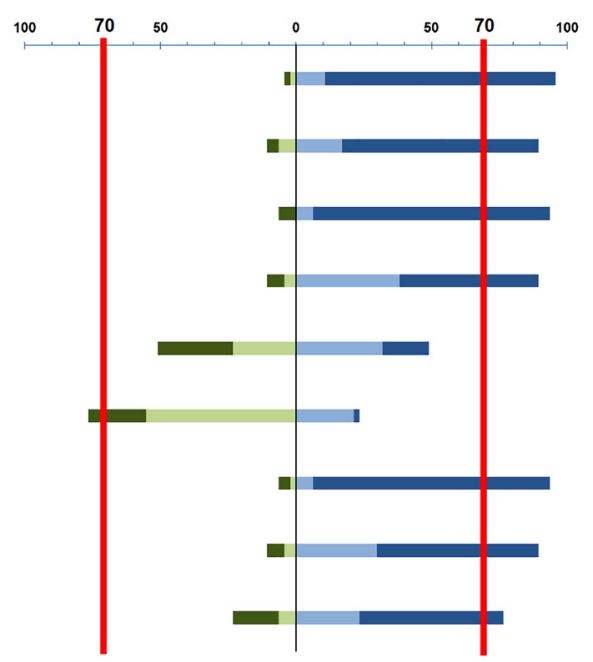

Is not indicated Should not be indicated
For caregivers with low risk of exposure

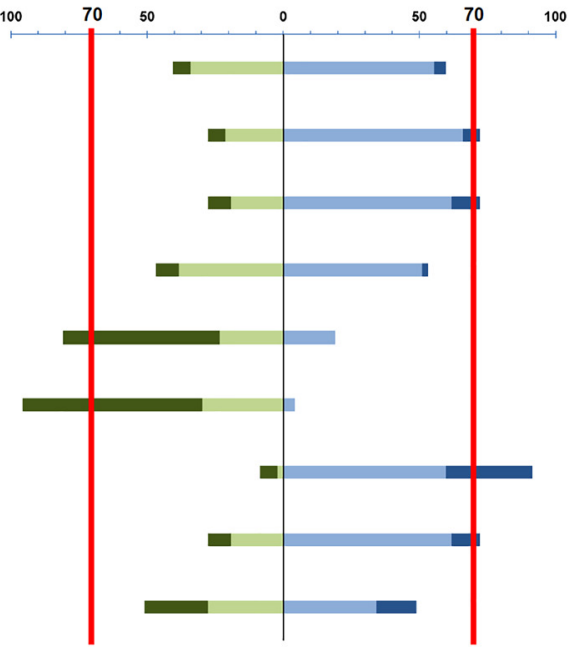

Could be indicated

Figure 2: Consensus for PPE for the prevention of exposure to liquid or aerosolized chemotherapy agents.

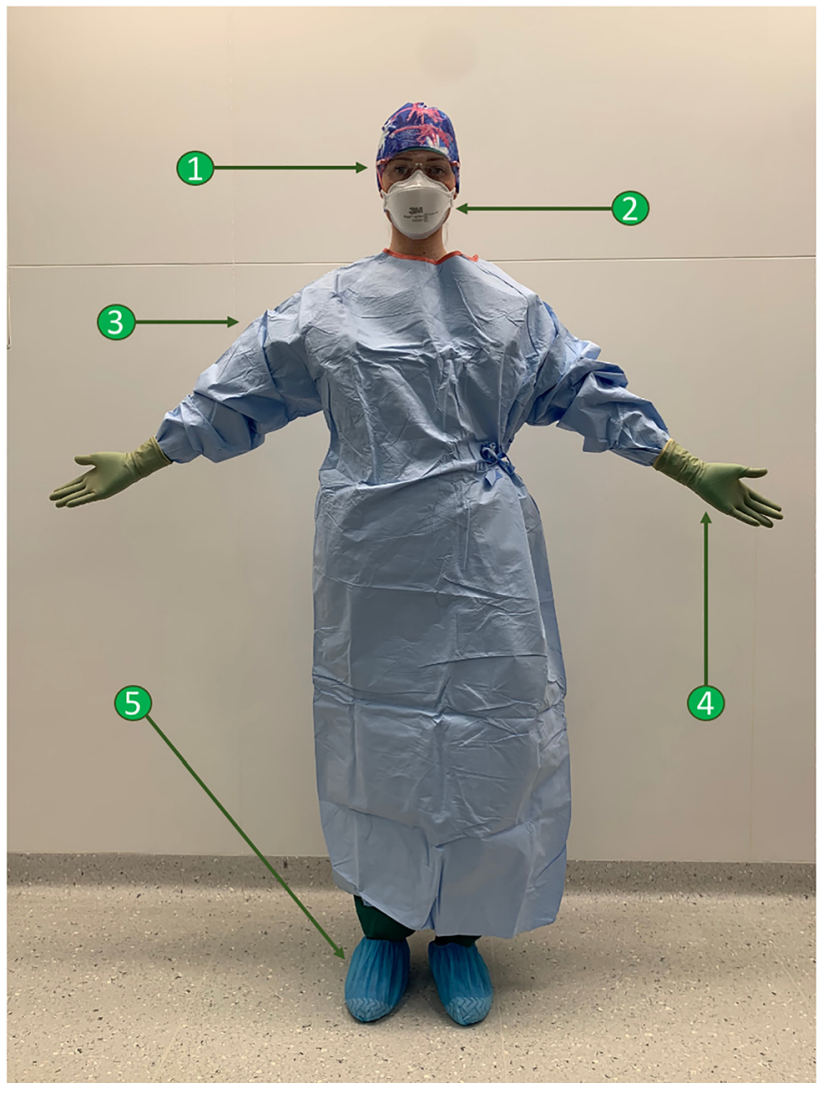

Figure 3: Recommended PPE during PIPAC procedure for caregivers at high risk of exposure.

Optimal PPE for caregivers at high risk of exposure during PIPAC: (1) Specific ocular protection (plastic goggles or eye shields), (2) FFP mask class 3, (3) reinforced surgical gown, (4) double gloving with an inner pair resistant to chemotherapy agents, and (5) plastic overshoes. the seven items evaluated regarding environmental protection, a strong positive recommendation was found in five, and their use should be routinely indicated: absorbent mats (95.7\% of agreement), labeled container under the injector head (95.7\%), transparent cover sheet (89.4\%), "en bloc" removal (93.6\%), and dedicated labeled waste containers (97.9\%). The use of a disposable cover over the injector monitor met a weak positive recommendation (72.3\%) and could be indicated. Strong negative recommendation (93.6\%) was found for the use of single-use laparoscopic camera and is therefore not indicated.

The original PIPAC safety protocol [4] recommends positioning a watertight drape on the floor and placing a waste bin for chemicals beneath the angioinjector head. Disposable covers on the injector monitor during PIPAC could help prevent the transfer of potential contamination to other surfaces [6]. After line disconnection incidents between the syringe and the high-pressure line, an additional recommendation was to protect the high-pressure line with a sterile plastic bag [16]. The aerosolizer, the line, and the syringe must be disposed of as a whole. One multicenter study [6] demonstrated a decrease in local contamination when surgical disposables were removed "en bloc" compared to removal after disconnection [24]. In order not to endanger third parties unnecessarily, every single-use material must be collected immediately by the surgical staff (wearing PPE) in specially designed and labeled waste containers. This waste material includes, among other things: empties (syringes, infusion containers, and lines), single-use instruments (e.g., trocars, aerosolizing device, etc.), operating drapes and gauzes, stitches, and needles. 
10. Use of absorbent mats on the floor

11. Placing a labeled container under the injector head to contain spillage

12. Wrapping a transparent cover sheet around the highpressure line to contain spillage

13. En-bloc removal of contaminated disposables into the labeled containers

14. Use of dedicated, sealed and labeled chemotherapy waste containers

15. Use of low-price single-use laparoscopic cameras

16. Use of disposable covers on the injector monitor

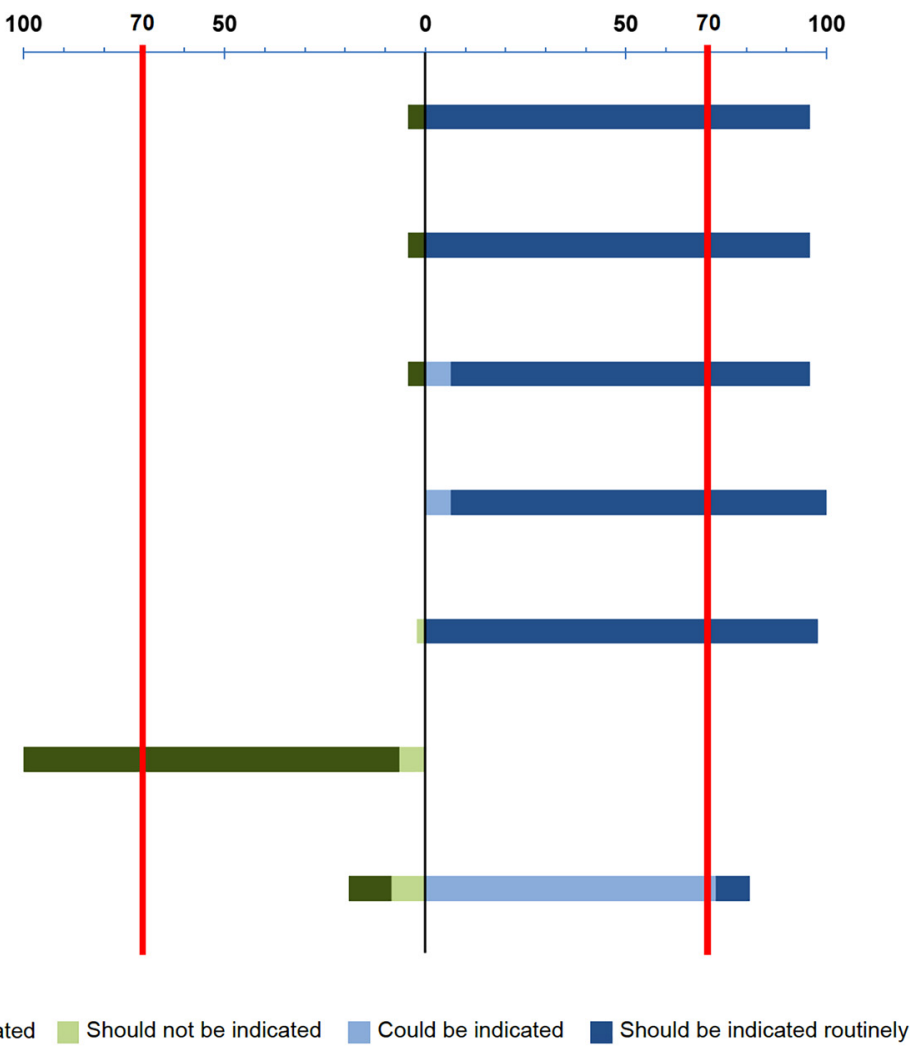

Figure 4: Consensus for environmental protection.

The watertight waste containers should be labeled in accordance with the dangerous goods and waste legislation, stating the nature of the waste - "cytotoxic and cytostatic waste", the UN number under dangerous goods law, and the hospital (sender) address. The hermetically closed containers are to be hand over undamaged to the disposal company. The laparoscopic camera (precisely: the Hopkins optics) is the only multi-use instrument exposed to CA. One study [12] demonstrated minimal traces of platinum on $1 / 3$ Hopkins optics directly after PIPAC, but not after the sterilization process. This shows that laparoscopic cameras can be safely reused after PIPAC.

\section{Prevention of exposure to aerosolized CA}

The degree of consensus for the different measures for preventing exposure to aerosolized CA is given in Figure 5. Eight items evaluated the prevention of exposure to aerosolized CA. The strong positive recommendation was found in six and should be routinely indicated: use of disposable balloon trocars (93.6\% of agreement), airtight pneumoperitoneum (100\%), advanced OR ventilation system (91.5\%), remote chemotherapy administration (95.7\%), remote video monitoring of aerosolization (89.4\%), and safe toxic aerosol evacuation (97.9\%). Two remaining items met weak positive recommendation, and therefore could be indicated: laminar airflow activation (48.9\%), and additional plastic cover protection with smoke filtration ("French system") (55.3\%).

A hazard specific to PIPAC is a potential inhalation exposure caused by possible CA leakage during administration [4]. A number of studies have investigated OR air contamination with cisplatin by measuring its concentration in air samples: none of the measurements performed in Germany (Bielefeld [4], Herne [11], Tübingen [6], Regensburg [6], and Leipzig [15]), France (Lyon, Strasbourg [14]), Belgium (Gent [9]), Denmark (Odense [7]), and The Netherlands (Eindhoven [25]) showed traces of platin in the air. The field study of Ametbischler et al., conducted under routine conditions at two different centers (Tübingen and Regensburg), is quantitatively (14 PIPAC procedures) and qualitatively (platin detection limit of $0.000000000003 \mathrm{~g}$ platin in 1,000 L air). Together, all these studies provide solid evidence that the three-level confinement system implemented for PIPAC effectively prevents inhalation exposure to toxic aerosols. 
17. Use of single-use trocars with balloon and extra-abdominal obturators

18. Control of an air-tight pneumoperitoneum before start of the CA aerosolization

19. Activation of an advanced OR ventilation system

20. Activation of laminar air flow

21. Additional use of a transparent plastic cover protection connected to a smoke filtration device

22. Remote administration of $C A$ with all $O R$ team members leaving the OR

23. Remote video monitoring (fixed camera, outside screen)

24. Evacuation of the toxic aerosol via a closed system (CAWS) including microparticle filters / surgical smoke evacuation system

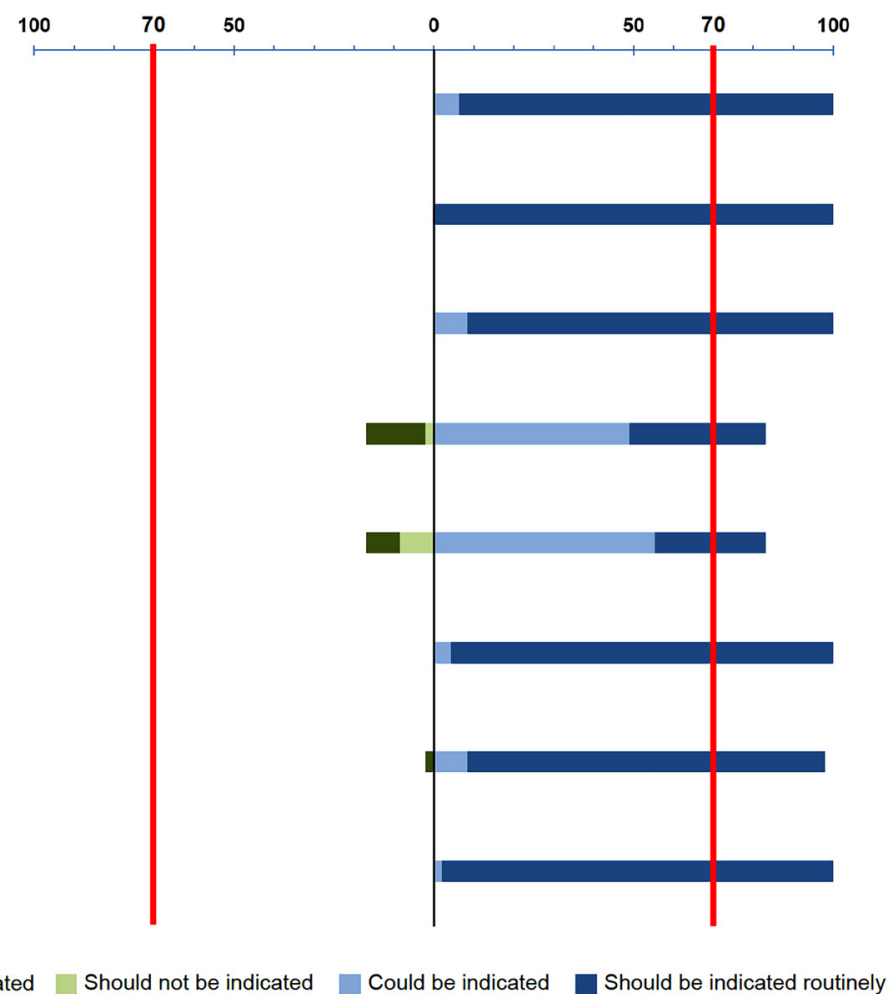

Figure 5: Consensus for prevention of exposure to aerosolized chemotherapy agents.

\section{General preventive measures}

High degree of consensus was reached for the use of general preventive measures (Figure 6). Access to the OR during PIPAC procedures is restricted in most centers, and the OR is usually labeled as a hazard area. The original PIPAC safety checklist [4] is widely adopted, with some minor local adaptations. This safety list is advocated in ISSPP training modules [22]. Emergency kits are broadly available, including absorbent devices, mild soap, bleach, and eyewash kit, for quick CA absorption and first medical aid [26]. Pregnant women should not participate in PIPAC procedures, in analogy to hyperthermic intraperitoneal chemotherapy (HIPEC) procedures [26, 27]. Platinum contamination on the floor of the OR has been detected up to 3 days after the HIPEC procedure, underlining the importance of effective cleaning methods [28]. In contrast, floor contamination is barely observed after PIPAC. However, a significant, in some cases, high surface contamination of the angioinjector has been documented after PIPAC, suggesting leakage during syringe manipulation and connection, as well as insufficient cleaning methods. In one study [6], contamination was higher before PIPAC as compared to after PIPAC, while another study [8] showed that contamination remained after cleaning. These results imply that the cleaning of the angioinjector has received insufficient attention so far. A revised cleaning method with triple-wiping was reported to reduce injector contamination [8].

In this Delphi study, the need for information and training was estimated to be high (Table 1). A recent survey amongst PIPAC expert centers showed a rather low rate of adhesion to protection measures. Safety awareness did not reach expected levels for a highly standardized procedure, with a lower information score among anesthesiologists and cleaning staff. Availability of emergency kits in case of accidental exposure was unknown for $50 \%$ of responders. Most OR team members seek supplementary information about the risks related to CA administration [18]. Another recent study demonstrated that nonmedical caregivers in the OR are aware of the occupational hazards related to the use of CA. However, there is a high need for continuous education for the healthcare personnel participating in PIPAC procedures [19]. 
Table 1: Consensus on the need of information and training.

\begin{tabular}{|c|c|c|c|c|}
\hline & Surgeons & Scurb Nurses & Anesthesiology team & $\begin{array}{r}\text { Cleaning Staff } \\
\\
\end{array}$ \\
\hline $\begin{array}{l}\text { Prior information related to } \\
\text { biohazards of administration of CA }\end{array}$ & $97.9 \%$ & $95.7 \%$ & $91.5 \%$ & $87.2 \%$ \\
\hline Prior training on safety measures & $95.7 \%$ & $93.6 \%$ & $89.4 \%$ & $78.7 \%$ \\
\hline $\begin{array}{l}\text { Prior information on emergency } \\
\text { measures }\end{array}$ & $97.9 \%$ & $95.7 \%$ & $95.7 \%$ & $83 \%$ \\
\hline $\begin{array}{l}\text { Prior information on waste } \\
\text { management and cleaning } \\
\text { procedures }\end{array}$ & $95.7 \%$ & $95.7 \%$ & $72.3 \%$ & $93.6 \%$ \\
\hline
\end{tabular}

Data outlined as percentage of strong positive recommendation.

25. During PIPAC procedure, labeled entrance of the OR instead of standard OR policy

26. During PIPAC procedure, restricted access for all personnel not directly involved (including students or visitors, for instance) instead of standard OR policy

27. During PIPAC procedure, the use of a checklist before aerosolization to double-check all mandatory safety components

28. During PIPAC procedure, the availability of an emergency kit for accidental exposure to chemotherapeutic agents, and ready to use in the OR

29. Extended injector cleaning procedure (triple wiping of the PIPAC injector) to reduce risk of recurrent OR contamination, compared to standard cleaning procedure

30. Access to PIPAC procedure for pregnant collaborators* tLabelled answerd:

Is not permitted; Should not be permitted; Could be permitted; Should be permitted

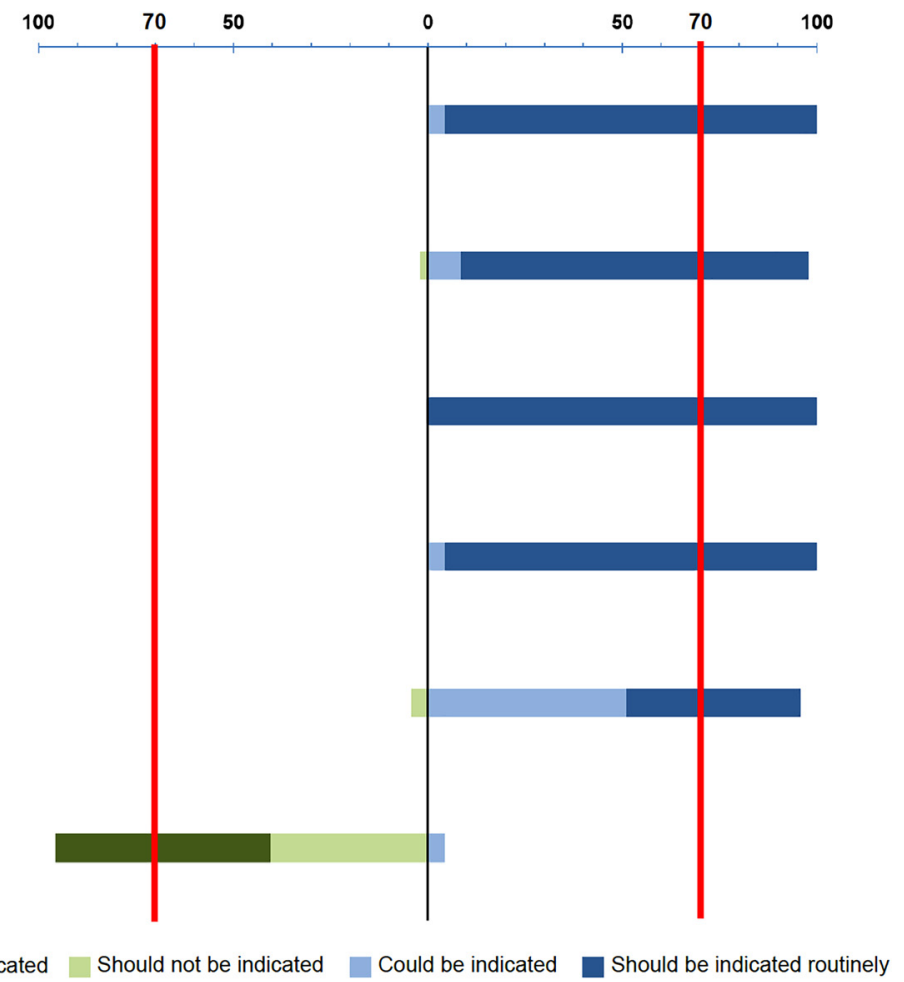

Figure 6: Consensus for general preventive measures. 


\section{Discussion}

In this Delphi study, a high degree of consensus was reached for a comprehensive safety protocol for PIPAC, adapted to the risk of exposure for the different caregivers in the OR.

Little is known about incidents or safety breaches during PIPAC procedures. All existing safety protocols have been adapted from the original protocol from the pioneer team in Germany which had been developed under the auspices of German regulatory bodies with a strong focus on healthcare safety. Early experience from Lausanne [22], reported only minor events during aerosolization, i.e. automatic stop of the injector due to pressure limitation and minor chemotherapy leaks, entirely confined by the cover sheet systematically used. Direct incidental exposure of the OR co-workers, has not been described [10]. In a recent survey assessing safety perception of intraperitoneal chemotherapy administration (PIPAC and HIPEC) from 211 OR co-workers [18], 28\% reported being aware of at least one incident, without further details. Prospective multicentric auditing of safety breaches during PIPAC procedure might provide more detailed information on the subject in the future.

However, over the years, safety protocols have been slightly adapted, most likely due to physician preference on certain aspects and safety material availability, leading to some variations between different countries. In our study was not possible to reach a sufficient degree of consensus for the following measures:

\section{Laminar airflow}

Although laminar airflow was considered mandatory in the initial risk evaluation, several independent studies showed that laminar airflow is not needed. An advanced OR ventilation system meeting the norm ISO $14644-1$ class $\leq 5$ is sufficient to prevent inhalation exposition during PIPAC $[4,6,7$, $9,11,15]$. The French PIPAC centers developed an alternative protocol using a plastic drape covering the patient and tubing connected to a mobile HEPA filtering device [15]. This protocol was audited successfully by the national safety authority in France (INRS - Institut National de Recherche et Sécurité) [8, 29]. The alternative "French" system is currently widely used in France and other countries. In a study on two PIPAC procedures, no platin traces were measured in the air with a sensitivity of $<0.00000002 \mathrm{~g} / \mathrm{m}^{3}$ [14]. In spite of the supportive evidence available for this system, the German Worker's insurance does not allow PIPAC performance in OR with no advanced ventilation/filtration system.

\section{FFP masks}

Wearing FFP mask class 2 (filtering 94\% of particles with a diameter between 0.01 and $1 \mu \mathrm{m}$ ) or class 3 (filtering $99 \%$ of particles) offers additional protection against inhalation exposition to toxic aerosols during PIPAC. On the other side, such masks were not recommended in the initial risk evaluation, assuming the $\mathrm{OR}$ ventilation/filtration system could reduce the inhalation risk to an acceptable minimal level. Numerous negative air measurements (see above) confirmed that the person's safety during PIPAC is not dependent on wearing FFP class 2 or 3 masks. On the other side, the German workers' insurance recommends wearing FFP-3 masks during HIPEC [30] and, in analogy, it appears reasonable to propose FFP class 2 or 3 masks as an additional protective measure.

\section{Surface contamination}

The evidence available shows that there can be surface contamination (in particular of the angio-injector) after PIPAC. An important lesson from Ametbischler's field study [6] is that the surface contamination varies by four orders of magnitude (=10.000 times) between individual PIPAC procedures. In some PIPAC procedures, surface contamination is absent after careful handling of the chemotherapy syringes and qualified instrument operation. In other procedures, significant contamination was detected, in some cases even high levels of contamination, probably following unqualified handling. These findings underline

- The need for adequate, repeated, documented training of the persons operating the angio-injector during PIPAC, in order to guarantee proper handling. It is legally and medically inacceptable to allow unqualified personal to operate the angioinjector.

- The need for adequate cleaning of the angioinjector after the procedure, through qualified personal wearing proper PPE.

There might be methodological issues with some studies, showing e.g. surface contamination before but not after PIPAC, or floor contamination in spite of the presence of a drape on the floor and with no air contaminations. Such findings suggest that chemotherapy traces were present before PIPAC, e.g. when PIPAC is performed in a room regularly used for HIPEC procedures handling larger volumes of chemotherapeutic solutions.

Taken together, this Delphi study shows a large consensus between PIPAC centers on most safety measures during PIPAC. Some practices differ, such as the use of a 
plastic cover drape with a mobile HEPA filtering system, or wearing FFP masks. This Delphi study is meant to support hospital management, physicians, nurses, and regulatory authorities in making decisions concerning appropriate safety measures during PIPAC. The systematic statement developed in this study reflect the current opinion of surgeons, anesthesia, and nurses on safety measures needed during PIPAC procedures, and do not reflect the opinion of the ISSPP.

The EC guidelines, in particular Directive 89/391/EEC, lay down the main principles to encourage improvement in the safety and health of workers at work. These principles are precised in further directives, e.g. 2004/37/EC "carcinogens or mutagens at work" and 2019/1831 "indicative occupational exposure limit values". These directives have been translated into national laws and regulations, which might differ slightly between countries, and also between the EC and other locations in the world. In the EC, it is the responsibility of the employers to take the measures required by these laws and regulations.

In summary, a high degree of consensus was reached for a comprehensive and risk-adapted safety protocol for PIPAC for the different caregivers in the OR. This consensus can be a common basis for education and implementation and provide valuable guidance helping to reach high adherence and a safe procedure.

Research funding: None declared.

Author contributions: All authors have accepted responsibility for the entire content of this manuscript and approved its submission.

Competing interests: Authors state no conflict of interest. Informed consent: Not applicable.

Ethical approval: Not applicable.

\section{Appendix}

\section{ISSPP PIPAC study group}

Julio Abba (Digestive Surgery, University Hospital Greno ble Alpes, Grenoble, France); Adnane Afifi (Surgical Oncology, Casablanca, Marocco); Michael Bau Mortensen (Department of Surgery, Odense Pancreas Center [OPAC], Odense University Hospital, Odense, Denmark); G. Bharath (Department of Surgical Oncology, Malleswaram, Bangalore, India); Aditi Bhatt (Department of Surgical Oncology, Zydus Hospital, Ahmedabad, India); Jimmy Bok Yan So (Department of Surgery, National University of Singapore, Yong Loo Lin School of Medicine, Singapore, Singapore); Andreas Brandl
(Digestive Unit, Champalimaud Foundation, Lisbon, Portugal); Wim Ceelen (Department of GI Surgery and Cancer Research Institute Ghent [CRIG], Ghent University Hospital, Ghent, Belgium); Delia Cortes-Guiral (Department of Colorectal Surgery, King Khalid Hospital, Nejran, Saudi Arabia); Thomas Courvoiser (Department of Digestive Surgery, CHU Poitiers, Poitiers, France); Julien Coget (Department of Digestive Surgery, CHU Rouen, Rouen, France); Ignace $\mathrm{H}$. de Hingh (Department of Surgery, Catharina Hospital, Eindhoven, the Netherlands); JeanBaptiste Delhorme (Department of Digestive Surgery, CHU Hautepierre, Strasbourg, France); Suryanarayana S. V. Deo (Department of Surgical Oncology, Dr BRA IRCH, AIIMS, New Delhi 110029, India); Andrea di Giorgio (Department of Digestive Surgery, Fondazione Policlinico Universitario Agostino Gemelli IRCCS, Rome, Italy); Frederic Dumont (Department of Surgical Oncology, Institut Cancérologique de l'Ouest, Saint Herblain, France); Cecilia Escayola (Division of Gynaecologic Surgery, Clinica del Pilar, Barcelona, Spain); Anne-Cécile Ezanno (Department of Surgery, HIA Begin, Saint Mandé, France); Johan Gagnière (Department of Hepatobiliary and digestive surgery, CHU Estaing, Clermont-Ferrand, France); Julio Galindo (Department of General and Digestive Surgery, Hospital Universitario Ramón y Cajal, Madrid, Spain); Torben Glatz (Department of General and Visceral Surgery, Medical Center - University of Freiburg, Freiburg, Germany); Tarkan Jäger (Department of Surgery, Paracelsus Medical University, Salzburg, Austria); Maximilian Jarra (Department of General Surgery, Campus Virchow Klinikum, Charité, Universitätsmedizin Berlin, Berlin, Germany); Ninad Katdare (Department of General Oncology, Sir H. N. Reliance Foundation Hospital and Research Centre, Mumbai, India); Vahan Kepenekian (Department of Digestive Surgery, Centre Hospitalier Lyon Sud, Lyon, France); Vladimir M. Khomyakov (Department of thoracoabdominal cancer surgery, P.A. Hertsen Moscow Oncology Research Center, Moscow, Russia); Konstantinos Kothonidis (Department of Digestive Surgery, CHR Val de Sambre, Sambreville, Belgium); Nathalie Laplace (Department of Digestive Surgery, Centre Hospitalier Lyon Sud, Lyon, France); Vincent Lavoue (Department of Gynecology, CHU Rennes, Rennes, France); Kuno Lehmann (Department of Surgery and Transplantation, Univer sity Hospital of Zurich, Zurich, Switzerland); Craig Lynch (Division of Cancer Surgery, Peter MacCallum Cancer Centre, Melbourne, Victoria, Australia); Sanket Mehta (Depart ment of Surgical Oncology, Saifee Hospital, Mumbai, India); Bogdan Moldovan (Department of General Surgery, "Sf. Constantin” Private Hospital Braşov, Romania); Aviram Nissan (Department of General and Oncological SurgerySurgery C, The Chaim Sheba Medical Center, Tel Hashomer, Ramat Gan, Israel); Maciej Nowacki (Department of Surgical 
Oncology, Ludwik Rydygier's Collegium Medicum, Nicolaus Copernicus University in Torun, Bydgoszcz, Poland); David Orry (Department of Surgical Oncology, Centre Geor ges-François Leclerc, Dijon, France); Gloria Ortega Pérez (Department of Surgical Oncology, MD Anderson, Madrid, Spain); Urs G. Pabst (Department of Surgery, Ruhr University Bochum, Bochum, Germany); Brice Paquette (Department of Digestive Surgery, CHU Jean Minjoz, Besançon, France); Marius Paskonis (Centre of Abdominal Surgery, Vilnius University Hospital Santariškiu Klinikos, Vilnius, Lithuania); Pompiliu Piso (Department of General and Visceral Surgery, Barmherzige Brueder Hospital Regensburg, Regensburg, Germany); Marc Pocard (Department of Digestive and Visceral Surgery, APHP Lariboisière, Paris); Beate Rau (Department of Surgery, Campus Virchow-Klinikum and Charité Campus Mitte, Charité-Universitätsmedizin Ber lin, Germany); Marc Reymond (Department of Surgery, University of Tübingen, Tübingen, Germany); Frederic Ris (Division of Digestive Surgery, University Hospitals of Geneva, Geneva, Switzerland); Manuela Robella (Unit of Surgical Oncology, Candiolo Cancer Institute-FPO, IRCCS, Turin, Italy); José Silvestre-Rodriguez (Department of General Surgery, Hospital Universitario de Gran Canaria Dr. Negrin, Las Palmas de Gran Canaria, Spain); Shivendra Singh (Department of Gastro-intestinal Oncosurgery \& Liver Transplantation, Rajiv Gandhi Cancer Institute, New Delhi, India); S. P. Somashekhar (Department of Surgical Oncology, Manipal Comprehensive Cancer Center, Manipal Hospital, Bangalore, India); Claudio Soravia (Laparoscopic Robotic Surgery, Clinique Générale-Beaulieu, Geneva, Switzerland); Isabelle Sourrouille (Department of Surgical Oncology, Institut Gustave Roussy, Villejuif, France); Abelkader Taibi (Department of Digestive Surgery, CHU Dupuyren, Limo ges, France); Clemens Tempfer (Department of Obstet rics and Gynecology, Ruhr-Universität Bochum, Bochum, Germany); Jared Torkington (Department of General Surgery, University Hospital of Wales, Cardiff, United Kingdom); Giuseppe Vizzielli (Division of Gynecologic Oncology, Catholic University of the Sacred Heart, Rome, Italy); Wouter Willaert (Department of GI Surgery and Cancer Research Institute Ghent [CRIG], Ghent University Hospital, Ghent, Belgium).

\section{References}

1. Alyami M, Hübner M, Grass F, Bakrin N, Villeneuve L, Laplace N, et al. Pressurised intraperitoneal aerosol chemotherapy: rationale, evidence, and potential indications. Lancet Oncol 2019;20:368-77.
2. Grass F, Vuagniaux A, Teixeira-Farinha H, Lehmann K, Demartines $\mathrm{N}$, Hübner $M$. Systematic review of pressurized intraperitoneal aerosol chemotherapy for the treatment of advanced peritoneal carcinomatosis. Br J Surg 2017;104:669-78.

3. Solass W, Kerb R, Mürdter T, Giger-Pabst U, Strumberg D, Tempfer C, et al. Intraperitoneal chemotherapy of peritoneal carcinomatosis using pressurized aerosol as an alternative to liquid solution: first evidence for efficacy. Ann Surg Oncol 2014;21:553-9.

4. Solass W, Giger-Pabst U, Zieren J, Reymond MA. Pressurized intraperitoneal aerosol chemotherapy (PIPAC): occupational health and safety aspects. Ann Surg Oncol 2013;20:3504-11.

5. Reymond L, Solass W, Tempfer CB, Reymond MA. Pressurized Intraperitoneal Aerosol Chemotherapy (PIPAC): occupational health and safety management. In: Berhart LV, editor. Advances in medicine and biology. New York: Nova Science Publishers; 2015:157-80 pp.

6. Ametsbichler P, Böhlandt A, Nowak D, Schierl R. Occupational exposure to cisplatin/oxaliplatin during Pressurized Intraperitoneal Aerosol Chemotherapy (PIPAC)? Eur J Surg Oncol 2018;44:1793-9.

7. Graversen M, Pedersen PB, Mortensen MB. Environmental safety during the administration of pressurized IntraPeritoneal Aerosol Chemotherapy (PIPAC). Pleura Peritoneum 2016;1:203-8.

8. Ndaw S, Hanser O, Kenepekian V, Vidal M, Melczer M, Remy A, et al. Occupational exposure to platinum drugs during intraperitoneal chemotherapy. Biomonitoring and surface contamination. Toxicol Lett 2018;298:171-6.

9. Willaert W, Sessink P, Ceelen W. Occupational safety of pressurized intraperitoneal aerosol chemotherapy (PIPAC). Pleura Peritoneum 2017;2:121-8.

10. Rouche A, Hübner M, Grass F, Pache B, Demartines N, Blanc C. Anaesthesia in a toxic environment: pressurised intraperitoneal aerosol chemotherapy: a retrospective analysis. Turk J Anaesthesiol Reanim 2020;48:273-9.

11. Oyais A, Solass W, Zieren J, Reymond MA, Giger-Pabst U. Occupational health aspects of pressurised intraperitoneal aerosol chemotherapy (PIPAC): confirmation of harmlessness. Zentralbl Chir 2016;141:421-4.

12. Demtroder C, Tophoven G, Zieren J, Tempfer C, Reymond MA. Platinum contamination of laparoscopic instruments during pressurized intra peritoneal aerosol chemotherapy (PIPAC). J Pharm Pharmaceut Sci 2016;5:8-11.

13. Jansen-Winkeln B, Thieme R, Haase L, Niebisch S, Pommer C, Lyros $\mathrm{O}$, et al. Perioperative safety of intraperitoneal aerosol chemotherapy: analysis of our first 111 pressurized intraperitoneal aerosol chemotherapy (PIPAC) procedures. Chirurg 2019;90:137-45.

14. Delhorme JB, Klipfel A, D’Antonio F, Greget MC, Diemunsch P, Rohr S, et al. Occupational safety of pressurized intraperitoneal aerosol chemotherapy (PIPAC) in an operating room without laminar airflow. J Vis Surg 2019;156:485-8.

15. Cazauran JB, Alyami M, Lasseur A, Gybels I, Glehen O, Bakrin N. Pressurized intraperitoneal aerosol chemotherapy (PIPAC) procedure for non-resectable peritoneal carcinomatosis (with video). J Gastrointest Surg 2018;22:374-5.

16. Giger-Pabst U, Tempfer CB. How to perform safe and technically optimized pressurized intraperitoneal aerosol chemotherapy (PIPAC): experience after a consecutive series of 1200 procedures. J Gastrointest Surg 2018;22:2187-93. 
17. Alyami M, Sgarbura O, Khomyakov V, Horvath P, Vizzielli G, So J, et al. Standardizing training for pressurized intraperitoneal aerosol chemotherapy. Eur J Surg Oncol 2020;46:2270-5.

18. Clerc D, Hübner M, Ashwin KR, Somashekhar SP, Rau B, Ceelen W, et al. Current practice and perceptions of safety protocols for the use of intraperitoneal chemotherapy in the operating room: results of the IP-OR international survey. Pleura Peritoneum 2021;6:39-45.

19. Al Hosni M, Rouget C, Cusumano C, Garcia Lozcano E, Popescu H, Carrere S, et al. Non-medical caregivers and the use of intraperitoneal chemotherapy in the operating theatre: a survey on the perception of safety. J Vis Surg 2020;157:461-7.

20. Brindle M, Nelson G, Lobo DN, Ljungqvist O, Gustafsson UO. Recommendations from the ERAS ${ }^{\circledR}$ Society for standards for the development of enhanced recovery after surgery guidelines. BJS Open 2020;4:157-63.

21. Huang X, Lin J, Demner-Fushman D. Evaluation of PICO as a knowledge representation for clinical questions. AMIA Annu Symp Proc 2006;2006:359-63.

22. Hübner M, Grass F, Teixeira-Farinha $H$, Pache $B$, Mathevet $P$, Demartines N. Pressurized IntraPeritoneal Aerosol Chemotherapy - practical aspects. Eur J Surg Oncol 2017;43: 1102-9.

23. Andrews JC, Schünemann HJ, Oxman AD, Pottie K, Meerpohl JJ, Coello PA, et al. GRADE guidelines: 15. Going from evidence to recommendation-determinants of a recommendation's direction and strength. J Clin Epidemiol 2013;66:726-35.

24. Technische Regeln für Gefahrstoffe (TRGS) 525 , Gefahrstoffe in Einrichtungen der medizinischen Versorgung. GMBl; 2014. Available from: https://www.baua.de/DE/Angebote/ Rechtstexte-und-Technische-Regeln/Regelwerk/TRGS/pdf/ TRGS-525.pdf?_blob=publicationFile.
25. Rovers KP, Wassenaar ECE, Lurvink RJ, Creemers GM, Burger JWA, Los $M$, et al. Pressurized intraperitoneal aerosol chemotherapy (oxaliplatin) for unresectable colorectal peritoneal metastases: a multicenter, single-arm, phase II trial (CRC-PIPAC). Ann Surg Oncol 2021;28:5311-26.

26. Ferron G, Simon L, Guyon F, Glehen O, Goere D, Elias D, et al. BIG-RENAPE Working Group. Professional risks when carrying out cytoreductive surgery for peritoneal malignancy with hyperthermic intraperitoneal chemotherapy (HIPEC): a French multicentric survey. Eur J Surg Oncol 2015;41:1361-7.

27. Kyriazanos I, Kalles V, Stefanopoulos A, Spiliotis J, Mohamed F. Operating personnel safety during the administration of Hyperthermic Intraperitoneal Chemotherapy (HIPEC). Surg Oncol 2016;25:308-14.

28. Schierl R, Novotna J, Piso P, Böhlandt A, Nowak D. Low surface contamination by cis/oxaliplatin during hyperthermic intraperitoneal chemotherapy (HIPEC). Eur J Surg Oncol 2012;38: 88-94.

29. Passeron J, Guilleux A, Pilliere F. Prévenir les risques chimiques lors d'une chimiothérapie intrapéritonéale pressurisée par aérosolisation (CIPPA ou PIPAC). Ref Santé Trav 2016;147:29-39.

30. Heinemann A. Zytostatika im Gesundheitsdienst. Berufsgenossenschaft für Gesundheitsdienst und Wohlfahrtspflege (BGW); 2019. Available from: https://www. bgwonline.de/SharedDocs/Downloads/DE/Medientypen/BGW \%20Broschueren/BGW09-19-042_Zytostatika_im_ Gesundheitsdienst_Download.pdf?_blob=publicationFile.

Supplementary Material: The online version of this article offers supplementary material (https://doi.org/10.1515/pp-2021-0125). 